\title{
Equatorial modes observed in atmospheric variables
}

\author{
L KIRANMAYI and G S BHAT* \\ Center for Atmospheric and Oceanic Sciences, Indian Institute of Science, Bangalore 560 012, India. \\ *e-mail: bhat@caos.iisc.ernet.in
}

\begin{abstract}
Wavenumber-frequency spectral analysis of different atmospheric variables has been carried out using 25 years of data. The area considered is the tropical belt $25^{\circ} \mathrm{S}-25^{\circ} \mathrm{N}$. A combined FFTwavelet analysis method has been used for this purpose. Variables considered are outgoing longwave radiation (OLR), $850 \mathrm{hPa}$ divergence, zonal and meridional winds at 850,500 and $200 \mathrm{hPa}$ levels, sea level pressure and $850 \mathrm{hPa}$ geopotential height. It is shown that the spectra of different variables have some common properties, but each variable also has few features different from the rest. While Kelvin mode is prominent in OLR and zonal winds, it is not clearly observed in pressure and geopotential height fields; the latter two have a dominant wavenumber zero mode not seen in other variables except in meridional wind at $200 \mathrm{hPa}$ and $850 \mathrm{hPa}$ divergences. Different dominant modes in the tropics show significant variations on sub-seasonal time scales.
\end{abstract}

\section{Introduction}

The phenomena occurring on 20-80 day time scales in the tropics, known as the tropical intraseasonal variation (TISV), influence global weather and climate. The most dominant mode in TISV is the Madden Julian Oscillation (MJO) having time scale in 40-50 day period (Zhang 2005). Pioneering theoretical work by Matsuno (1966) on equatorial waves and spectral analysis of observed station data by Madden and Julian (1971) laid the foundation for a large number of studies to follow (Zhang 2005; Lau and Waliser 2005 for comprehensive reviews). Equations governing the atmospheric flow contain wave-like solutions and equatorial wave theory (EWT) forms the basis of much of the theoretical explanations for TISV (e.g., Wang 2005). In EWT, shallow water equations on the equatorial $\beta$-plane are solved, and the governing equations can be expressed as (e.g., Gill 1982; Wang 2005):

$$
\frac{\partial u}{\partial t}+\beta y v=-\frac{\partial \phi}{\partial x}+F_{x}+M(u)
$$

$$
\begin{aligned}
& \frac{\partial v}{\partial t}-\beta y u=-\frac{\partial \phi}{\partial y}+F_{y}+M(v) \\
& \frac{\partial u}{\partial x}+\frac{\partial v}{\partial y}+\frac{\partial \omega}{\partial p}=0
\end{aligned}
$$

where $t$ is time, $u, v$ and $\omega$ are respectively zonal, meridional and vertical (pressure) components of velocity, $\phi$ is geopotential height, $F_{x}$ and $F_{y}$ are frictional forces and $M(u)$ and $M(v)$ arise from the mean background flow. Assuming a wavelike solution (e.g., $\phi=\phi_{o} e^{i(k x-\nu t)}$, where $k$ and $\nu$ are wavenumber and frequency respectively and $i$ is the imaginary unit) and an equivalent depth $(h)$, theoretical dispersion relations are obtained. Equation (1) represents a set of coupled equations to be solved simultaneously, and the final solution takes into account all the dynamical interactions among the variables. For a given solution, all variables share the same $k, \nu$ and $h$.

The solutions consist of both symmetric and antisymmetric waves, and their dispersion relations depend on meridional mode number $(n)$ and $h$ (Gill 1982). Symmetric waves include eastward

Keywords. Tropical intraseasonal oscillation; equatorial modes; MJO; equatorial wave theory. 
moving Kelvin waves, odd number modes of westward moving Rossby waves and eastward and westward moving inertio gravity waves. Antisymmetric waves include even modes of westward moving Rossby waves and inertio gravity waves and mixed Rossby gravity (MRG) waves. In addition, Rossby-Haurwitz modes are observed at higher equivalent depth (Kasahara 1980; Madden 1978). Spectral analysis of atmospheric data in early 1970s did reveal certain preferred modes in the tropics (e.g., Yanai and Maruyama 1966; Wallace and Kousky 1968; Madden and Julian 1971), however, concrete evidence in support of EWT had to wait till 1999 (Wheeler and Kiladis 1999, WK99 henceforth). WK99 computed wavenumberfrequency $(k-\nu)$ spectra taking 18-year long outgoing longwave radiation (OLR) time series over the tropics $\left(15^{\circ} \mathrm{N}-15^{\circ} \mathrm{S}\right)$. The (red noise) normalized spectra revealed several peaks that coincided with theoretical modes predicted by EWT, in particular, Kelvin, Rossby and MRG waves. However, MJO, the most energetic mode, does not directly fall on any standard theoretical curve, and it has been suggested that MJO could be moist Kelvin wave (e.g., Wang 2005). WK99 also explored similarities and differences between OLR spectra and cross spectra between OLR, tropospheric temperature and geopotential height. It was shown that equivalent depths differ between OLR $(25 \mathrm{~m})$ and other variables $(200 \mathrm{~m})$. Cho et al (2004), Roundy and Frank (2004) and Masunaga (2007) also confirmed the existence of equatorial modes in OLR, precipitation and precipitable water data.

A majority of the studies where $k-\nu$ spectra are reported have analysed OLR, precipitation and precipitable water or show cross-spectra of OLR with zonal wind, temperature and geopotential height. Only exception is a recent study by Hendon and Wheeler (2008) where $k-\nu$ spectra of winds are shown. It is seen from equation (1) that OLR and precipitation are not the fundamental variables in EWT but $u, \nu, \phi$ are. In fact, OLR/precipitation is an end-product of several complex processes taking place in the atmosphere. In none of the previous studies, $k-\nu$ spectra (and corresponding $h$ ) of different variables have been shown or compared in one place.

Often, theoretical solutions are obtained under several simplifying assumptions while the real flow in the atmosphere is highly complex. Therefore, it is important to look at the spectra of the (observed) atmospheric variables and then examine the agreement with theory. For a given mode/solution, theory assumes the same values of $k, \nu$ and $h$ for all the variables and we expect some similarity in their spectra. Having said so, we like to add that few theoretical equatorial waves that are symmetric in some variables but antisymmetric in others. For example, an MRG wave is antisymmetric in geopotential and zonal wind, but symmetric in meridional wind (e.g., Wheeler 2002). Therefore, while the precise nature of the theoretical spectra may be variable and mode-dependent, the equivalent depths should be comparable. What is not clear from literature is how the spectra of different variables observed in the tropical atmosphere look like? Computing, comparing and contrasting $k-\nu$ spectra of OLR, $850 \mathrm{hPa}$ divergence, zonal $(U)$ and meridional $(V)$ winds, sea level pressure (SLP) and $850 \mathrm{hPa}$ geopotential height $\left(\phi_{850}\right)$ form the main objective of this paper.

Events in the atmosphere are not periodic but episodic and different equatorial modes have characteristic seasonal/geographic preferences (e.g., Wheeler et al 2000; Zhang and Dong 2004). Several studies have addressed this issue by employing band pass filtered data (e.g., Slingo et al 1996; Lawrence and Webster 2001; Teng and Wang 2003; Wheeler and Hendon 2004). For example, Slingo et al (1996) used running mean variance of 20-100 day filtered zonal mean $150 \mathrm{hPa}$ zonal wind to quantify TISV activity and study its interannual variations and relation to El Niño. Wheeler et al (2000) used wavenumber-frequency filtering of OLR data to obtain temporal variations in different modes by taking time series in the region where maximum variance in the mode of interest is observed. The structure, spatial and temporal distribution of different modes are presented using this method. Roundy and Frank (2004) presented climatological variations of different modes over northern and southern hemispheres separately using OLR and precipitable water data. Wheeler and Hendon (2004) devised an index for MJO from principal components of combined empirical orthogonal functions (EOFs) of zonal winds at $850 \mathrm{hPa}, 200 \mathrm{hPa}$ and OLR, and called it as real time multivariate MJO (RMM) index. The first two principal components (denoted by RMM1 and RMM2) represent $25 \%$ of total variance in MJO, and the sum of their squares $\left(\mathrm{RMM}^{2}+\mathrm{RMM} 2^{2}\right)$ is RMM. MJO is considered to be active if RMM exceeds 2. Using RMM, temporal and spatial evolution of MJO could be followed continuously in time. Masunaga (2007) presented regional and seasonal variations of MJO and its relationship with Kelvin and equatorial Rossby modes using seasonally averaged wavenumber-frequency spectra for four seasons. Masunaga (2007) also examined the meridional variations over three latitudinal belts namely $15^{\circ}-5^{\circ} \mathrm{S}, 5^{\circ} \mathrm{S}-5^{\circ} \mathrm{N}$ and $5^{\circ}-15^{\circ} \mathrm{N}$.

Previous studies have mainly focused on seasonal/temporal variations in MJO. Monthly climatologies of different TISV modes have not been reported, and computing this forms the second objective of the paper. A combined FFT-wavelet 
transform is used to quantify the temporal variations in different modes. It is shown that the spectra of different variables have some common properties, but each variable also has few features different from the rest. Almost all TISV modes show significant variations on sub-seasonal time scales. The paper is organized as follows. Section 2 describes the data and methodology, section 3 compares the spectral characteristics of different variables, and section 4 , the variations in spectral characteristics of OLR with space and time. Discussion and conclusions are given in section 5 .

\section{Data and methodology}

\subsection{Data}

Variables considered are OLR, $850 \mathrm{mb}$ divergence, SLP, $\phi_{850}$, zonal and meridional winds at 200,500 and $850 \mathrm{hPa}$ pressure levels $\left(U_{200}\right.$, $U_{500}$ and $U_{850}$ and $V_{200}, V_{500}$ and $V_{850}$ respectively). SLP, $\phi_{850}, U_{200}, U_{500}, U_{850}, V_{200}, V_{500}$ and $V_{850}$ are NCEP (National Centers for Environmental Prediction) reanalysis products (Kalnay et al 1996) and are downloaded from the website http://www.cdc.noaa.gov. The OLR data is taken from National Oceanic \& Atmospheric Administration, USA (NOAA, Liebmann and Smith 1996). $850 \mathrm{hPa}$ divergence is taken from ECMWF (European Centre for Medium range Weather Forecasting) ERA-40 reanalysis (Uppala et al 2005). NCEP does not give divergence as a product (it can be computed), whereas divergence is one of the products in ERA-40 dataset. ERA-40 wind field is as reputed as that from NCEP, and hence ERA-40 divergence data has been used here. NCEP and NOAA data are daily values with $2.5^{\circ} \times 2.5^{\circ}$ spatial resolution for the period January 1979 to December 2003 spanning twenty five years. ERA-40 data is available at the same spatial and temporal resolutions but only up to August 2002. Hence the divergence data is taken from January 1979 till August 2002.

The main focus in this study is on tropical atmospheric variations on time scales ranging from a few days to less than a season. Therefore, data is filtered to remove time scales beyond 120 days. Filtering is done in the following way. First, FFT of the entire time series is taken (after applying cosine tapering at the ends) and the filtered time series reconstructed by retaining coefficients corresponding to time scales less than 120 days. This filtered time series was compared with that obtained from Lancoz window cosine filter (e.g., Slingo et al 1996) in few cases and the two curves are observed to be visually indistinguishable from each other when plotted. We believe that the results here are not dependent on the method of filtering.

\subsection{Wavelet analysis}

Events in the atmosphere are episodic in nature and their intensity/amplitude varies both in space and time. Such variations in amplitudes corresponding to different modes with time and space cannot be resolved completely using the conventional FFT. For example, while we find significant energy in MJO mode in the spectra, when in time this mode occurred and the corresponding preferred frequencies cannot be known from standard FFT. Windowed Fourier Transform (Kaiser 1994; Torrence and Compo 1998) can give local frequency information of a signal, however, the range of scales that can be studied for a given window is limited. When data contains a wide range of frequencies and the predetermination of the range is not possible, this method fails (Kaiser 1994). Now, wavelet analysis is widely used for the study of episodic processes (e.g., Gu and Zhang 2001; Wong 2009). The continuous wavelet transform $W$ of a time series $X(t)$ at time $t_{0}$ and scale $\tau$ is given by the expression (e.g., Daubechies 1990; Rao and Bopardikar 1998):

$$
W\left(t_{0}, \tau\right)=\frac{1}{\sqrt{\tau}} \int_{-\infty}^{\infty} X(t) \psi^{*}\left(\frac{t-t_{0}}{\tau}\right) d t
$$

In equation $(2), \psi$ is the mother wavelet function and $\psi^{*}$ is its complex conjugate. The width of the wavelet is the distance from the center of the wavelet where the amplitude becomes $1 / e$ times the maximum amplitude. Wavelet function can be either real or complex. Complex wavelet functions can give both amplitude and phase information and hence more useful for studying wave propagations whereas the real wavelets can be used to identify the peaks. The shape of the wavelet should reflect the main features in the variable. The scale resolution of a wavelet is also important. The scale of the wavelet $\tau$ is not directly equal to the Fourier period and is dependent on the mother wavelet used (Torrence and Compo 1998). In the present analysis, complex Morlet wavelet is used as the mother wavelet function and is given by:

$$
\psi(t)=\frac{1}{\sqrt{\pi}} e^{2 i \pi t} e^{-t^{2} / 2},
$$

with $t$ replaced by $\left(t-t_{0}\right) / \tau$ when calculating the transform. Morlet wavelet is ideal for the 
study of wave-like patterns in atmosphere and has been used by many of the previous studies (e.g., Torrence and Compo 1998; Kailas and Narasimha 2000; Shanker and Nanjundiah 2003; Zhang and Dong 2004). For the Morlet wavelet, Fourier period is related to $\tau$ as $4 \pi \tau /\left[2 \pi+\sqrt{ }\left(2+4 \pi^{2}\right)\right]$ (Torrence and Compo 1998). The Fourier period is 0.99 times the wavelet scale $\tau$. Hence $\tau$ is taken as representing the period directly, and the corresponding frequency is $\nu=1 / \tau$. Initially we considered using a two-dimensional wavelet transform to calculate $k-\nu$ spectrum. A recent study by Wong (2009) has shown some of the usefulness and limitations of this method in exploring convectively coupled waves. Main limitation is at low wavenumbers and wavenumber zero modes. Previous studies have shown that most energy lies at low wavenumbers in the intraseasonal time scales (WK99; Zhang 2005). At scales corresponding to low wavenumbers, the wavelet extends beyond one period and the integration domain practically covers the entire longitudinal belt. Thus, using the wavelet analysis also, longitudinal variations cannot be truly localized at low wavenumbers. Therefore, the emphasis in this study has been given to temporal variations in different tropical modes. For this purpose, a combined FFT-wavelet spectral method is used. FFT is used in the wavenumber domain, which also overcomes the cone of influence (Torrence and Compo 1998) related issues at low wavenumbers. Such combined FFT and wavelet method was used earlier by Gu and Zhang (2001) to explore zonal and temporal variations of synoptic scale disturbances in intertropical convergence zone.

To obtain the spectra, FFT coefficients are first calculated in the spatial domain at each time step. These FFT coefficients are functions of wavenumber and time $t_{0}$. Then, for each wavenumber, time series of the FFT coefficients are subjected to wavelet transform giving wavelet coefficients for different $t_{0}$ and $\tau$. The resulting coefficients are functions of $t_{0}, k$ and $\tau$. As the length of time series is sufficiently long (9132 days) compared to the time scales under consideration (maximum 100 days), edge effect in time domain is negligible. This method enables the calculation of temporal variations in $\nu-k$ spectra and also reduces the computational efforts compared to the twodimensional wavelet analysis. Another advantage of this method is its ability in calculating coefficients at wavenumber zero (i.e., zonal average), which is not possible in case of two-dimensional wavelet analysis (e.g., Wong 2009).

$k$ and wavelength $(\lambda)$ are related by $\lambda=360 / k$ where $\lambda$ is in degrees. Owing to the spherical geometry of the earth, the two end-points are the same in zonal direction. Hence wavelengths corresponding to integer values of $k$ are only considered in this study. From the wavelet coefficients, the average spectrum for time range from $t_{1}$ to $t_{2}$ corresponding to frequency $\nu$ is calculated from the expression below (Torrence and Compo 1998),

$$
\overline{W^{2}}(\nu, k)=\frac{1}{\left(t_{2}-t_{1}\right)} \sum_{t_{0}=t_{1}}^{t_{0}=t_{2}}\left|W\left(t_{0}, \nu, k\right)\right|^{2}
$$

This can be extended in space by averaging the square of coefficients in $k$ space as well in the case of two-dimensional wavelet spectra. Average $W$ obtained for a given wavenumber at a given frequency is taken as the spectral intensity at that particular wavenumber and frequency.

\subsection{Spectral normalization}

When the raw spectrum is examined, it is observed that the energy is concentrated in certain regimes/modes. However, these modes may not be statistically significant while certain modes which are not prominent here could be significant owing to the fact that the background noise is not white for atmospheric variables (Torrence and Compo 1998; Wheeler and Kiladis 1999). In order to extract the modes which are significant, many of the previous studies have normalized the raw spectra with appropriate background spectrum (often only the normalized spectra are shown). Therefore, normalization of raw spectra with appropriate background noise spectrum is important. Most widely used background spectrum for climate data is the red noise (e.g., Torrence and Compo 1998; WK99; Masunaga 2007). Red noise is a function of frequency and is given by (Torrence and Compo 1998):

$$
P_{k}=\frac{1-\alpha^{2}}{1+\alpha^{2}+2 \alpha \cos (2 \pi \nu / N)}\left(\frac{2 \sigma^{2}}{N}\right),
$$

where $\sigma^{2}$ is variance, $N$ the number of data points, $\alpha$ is lag -1 autocorrelation and $\nu=0, \ldots, N / 2$ is the frequency. WK99 calculated the background spectrum for normalizing the spectra as follows. First the average of symmetric and antisymmetric spectra is taken. This resultant spectrum is smoothened repetitively in both wavenumber and frequency using 3 point smoothening with $1-2-1$ weighting. The smoothening is repeated till there is no significant change in the spectrum with further iterations. The resultant background spectra for OLR using this method and theoretical red noise formulations are similar in nature and results obtained by normalizing with either of these 


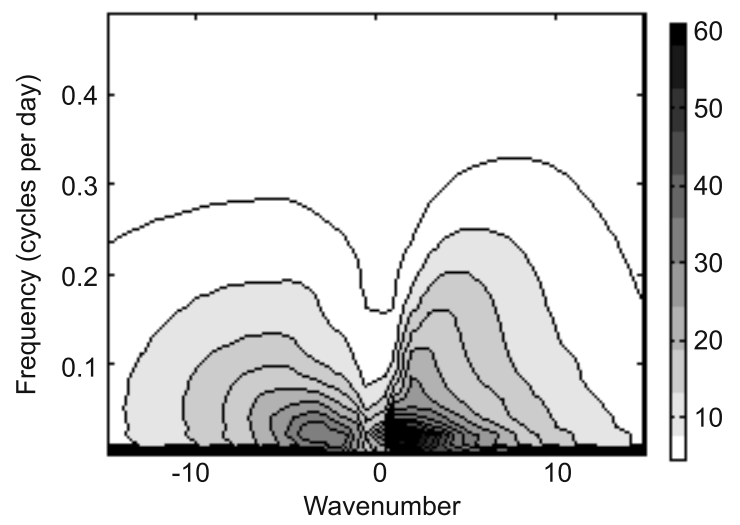

Figure 1. Wavenumber frequency spectrum of the symmetric part of OLR without normalization. The units are in $\left(\mathrm{W} \mathrm{m}{ }^{-2}\right) \mathrm{ms}$.

two are almost same (Hendon and Wheeler 2008). Hence in this work, results are presented using the background spectrum calculated following WK99.

The resulting normalized spectra with values above a certain threshold are considered significant at different levels of confidence. The significance test is carried out assuming chi square distribution for the spectral variations, with the null hypothesis that the spectra are not different from background noise. For the present time series, normalized spectral values above 1.1 are significant at $95 \%$ confidence. It is to be noted that the degrees of freedom for the filtered data are different from that of the raw data (e.g., Emery and Thomson 2001) and this fact is taken into account while calculating the significance level (Kiranmayi and Bhat 2008).

\section{Spectral characteristics}

To begin with, we show the time averaged raw and normalized spectra of OLR in figures 1 and 2 respectively. To facilitate the comparison of our results with those of WK99, average OLR spectra are calculated for same latitude belt, i.e., $15^{\circ} \mathrm{S}-15^{\circ} \mathrm{N}$. The spectra are shown for wavenumbers -15 to 15 and frequency 0.01 cycles per day (cpd) to $0.5 \mathrm{cpd}$. OLR is split into symmetric $\left(\mathrm{OLR}_{\text {sym }}\right)$ and antisymmetric $\left(\mathrm{OLR}_{\text {antisym }}\right)$ parts, where $\quad \operatorname{OLR}_{\text {sym }}=[\mathrm{OLR}(+$ lat. $)+\operatorname{OLR}(-$ lat. $)] / 2$ and $\operatorname{OLR}_{\text {antisym }}=[\mathrm{OLR}(+$ lat. $)-\operatorname{OLR}(-$ lat. $)] / 2$. Then the average from $15^{\circ} \mathrm{S}$ to $15^{\circ} \mathrm{N}$ is taken at every time step at each longitude. In the raw spectra (figure 1), more energy is contained in the eastward propagating modes with wavenumbers $1-3$ and time scale of more than 20 days [eastward moving intraseasonal (EISV) mode]. Low wavenumber low frequency westward propagating (Rossby) mode is also prominent. The normalized spectrum looks different (figure 2a) and here Kelvin mode emerges as one of the prominent modes. Relevant theoretical dispersion curves are shown in figure 2 for two equivalent depths, namely, $h=40 \mathrm{~m}$ and $75 \mathrm{~m}$. Spectral power is spread around the theoretical curves and reasonable matching is observed between theory and data for $h=40 \mathrm{~m}$ for the Kelvin mode. Maximum intensity in the normalized spectrum corresponds to EISV mode. Other peaks observed in symmetric spectrum are Kelvin mode, $n=1$ Rossby mode and TD (tropical depression) type variations. Antisymmetric part (figure 2b) includes EISV, MRG and $n=2$ Rossby modes. These observations are in good agreement with figure 3 of WK99. Thus the FFT-wavelet analysis method used in this study is robust and gives results consistent with those based on FFT. This has provided the confidence that the FFT-wavelet method can be used to study the features of TISV in more detail.

The next variable considered is $850 \mathrm{hPa}$ divergence. A negative value of low level divergence is associated with deep convection. Figure 3 shows the $k-\nu$ spectra for $850 \mathrm{hPa}$ divergence. It is seen that this variable has Kelvin and MRG modes in common with OLR but EISV and Rossby modes are nearly absent. $k=1$ mode stands out above noise level in EISV, that too barely. Another main difference is a prominent $k=0$ mode in the symmetric part in the divergence spectra.

Figure 4 shows the spectra of symmetric and antisymmetric parts of zonal winds. The wavenumber range shown in the spectra here on is limited to -10 to 10 as the spectral powers beyond this range are insignificant. Note that theoretical curve with $h=75 \mathrm{~m}$ fits the observed spectra better in case of $U_{850}$ and $h=40 \mathrm{~m}$ for $U_{200}$. For the symmetric part, spectra of $U_{850}, U_{500}$ and $U_{200}$ appear similar for the westward propagating $(k<0)$ modes, whereas for the eastward propagating modes the spectra of $U_{850}$ and $U_{200}$ are similar while that of $U_{500}$ is different. For $U_{500}$, the energetic mode in the eastward propagating wave regime is parallel to the Kelvin dispersion curve but shifted to the right in wavenumber space. In the antisymmetric part, spectra of $U_{850}$ and $U_{500}$ are similar for $k<0$ and that of $U_{850}$ and $U_{200}$ similar for $k>0$. When we examined the intensities in the symmetric spectrum, EISV mode is the most dominant mode at all the levels. Two high intensity bubbles at $k=-1$ and -4 and frequency around 0.2 day $^{-1}$ (period $\sim 5$ days) correspond to Rossby-Haurwitz mode. There is also another mode at $k=-1$ and having period around 15 days that lies between Rossby and Rossby-Haurwitz modes. In the antisymmetric part, MRG mode is weak or absent and EISV mode is seen at all the levels for $k=1$ (figure $4 \mathrm{~b}$ ).

Figure 5 shows the symmetric and antisymmetric parts of wavenumber-frequency spectra for 

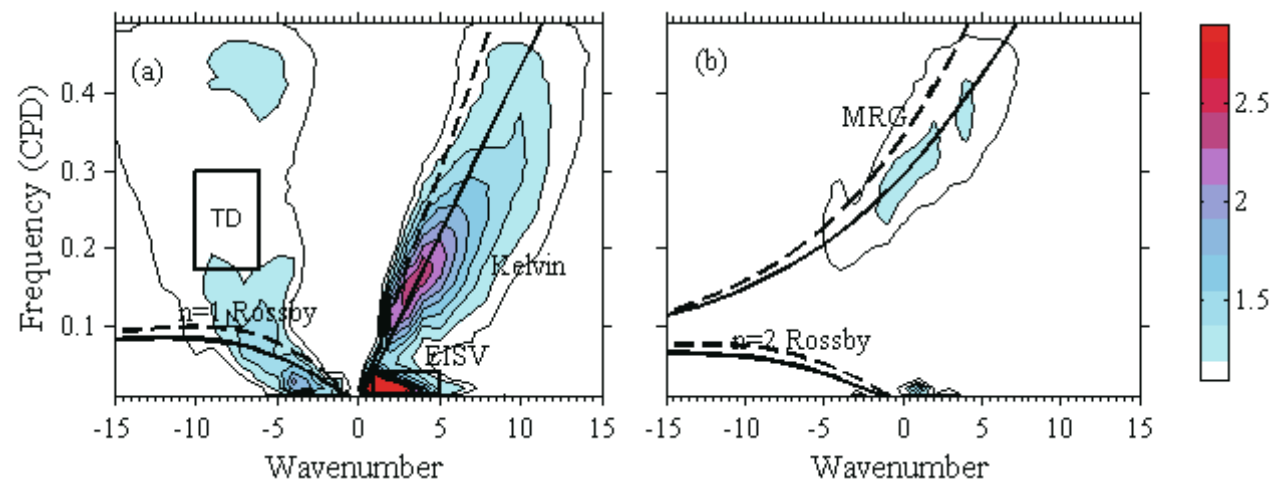

Figure 2. Wavenumber-frequency spectra of OLR averaged between $15^{\circ} \mathrm{N}$ and $15^{\circ} \mathrm{S}$ and normalized by the red noise. (a) Symmetric part and (b) antisymmetric part. The lines corresponding to the dispersion relations for different theoretical modes with $h=40 \mathrm{~m}$ (solid line) and $75 \mathrm{~m}$ (dashed line) are shown. The spectral regime corresponding to EISV and TD modes are shown in boxes in symmetric part.
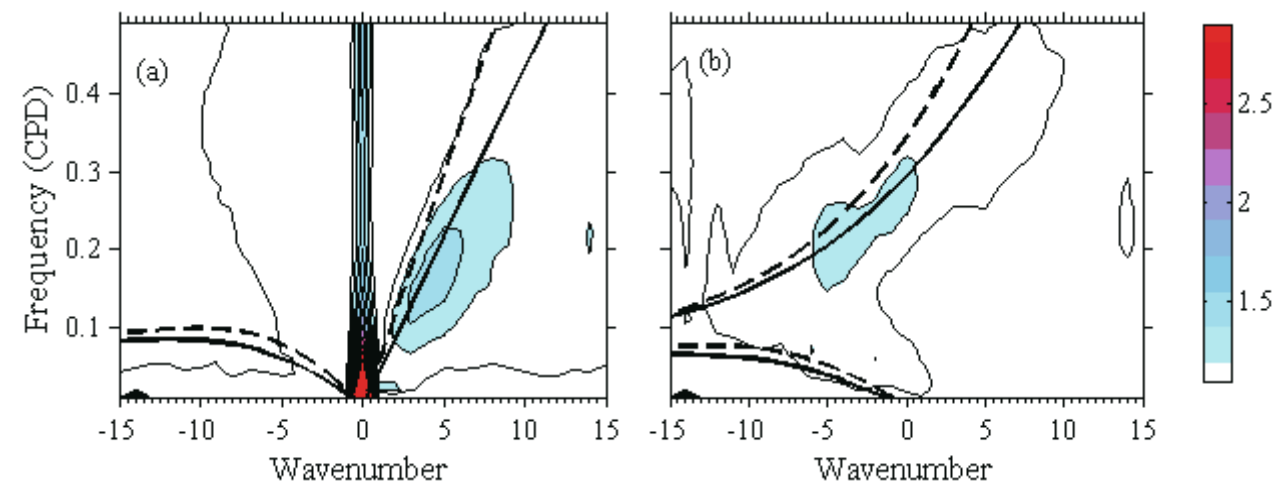

Figure 3. Same as figure 2, but for $850 \mathrm{hPa}$ divergence.

meridional winds. It has to be noted that modes in meridional winds have different symmetry compared to that of zonal winds (Wheeler 2002). The meridional winds in MRG, $n=$ even Rossby and Rossby-Haurwitz modes have symmetric structure whereas odd mode Rossby and Rossby-Haurwitz modes have antisymmetric structure. It is seen that the antisymmetric part doesn't have significant peaks except for Rossby-Haurwitz mode at $500 \mathrm{mb}$ level. Other features of the peaks are different from zonal winds. Westward MRG mode is observed for wavenumbers around -5 . As per EWT, meridional component of velocity is zero in Kelvin mode (Gill 1982). Therefore, the lack of spectral power in Kelvin mode is consistent with theory. Also the spectrum of $V_{200}$ has a peak in EISV regime which is absent at 850 and $500 \mathrm{hPa}$ levels. Another important feature observed in $V_{200}$ spectrum is the peak at wavenumber zero for all the frequencies. Also energy around Rossby-Haurwitz mode is present in $V_{200}$, and the peak shifts to higher (negative) wavenumbers towards MRG mode at other two levels. A peak at higher wavenumbers parallel to Kelvin mode as observed in $U_{500}$ is seen in meridional winds also.
Figure 6 shows the wavenumber-frequency spectra for SLP and $\phi_{850}$. (The antisymmetric parts are not shown as their spectral intensities are not significant.) Spectra of SLP and $\phi_{850}$ are somewhat similar and in contrast to OLR and zonal winds, low frequency zero wavenumber mode is most dominant here followed by $k=-1$ mode; dominant frequencies vary from less than 3 to 80 days. Kelvin mode is limited to low wavenumbers and importantly there is no clear Rossby mode.

\section{Temporal and spatial variations}

The results presented in section 3 represent average behaviour over a period of 25 years. In this section, average spectra for the individual months are discussed taking OLR as the variable. These spectra are obtained by averaging the coefficients corresponding to individual months, and normalizing the resulting value by the corresponding red noise (characterizing the entire period). The outcome of this exercise is shown in figure 7 . Both the areal extent (in $\nu-k$ space) and the intensity of different modes change from month to month clearly 
(a)

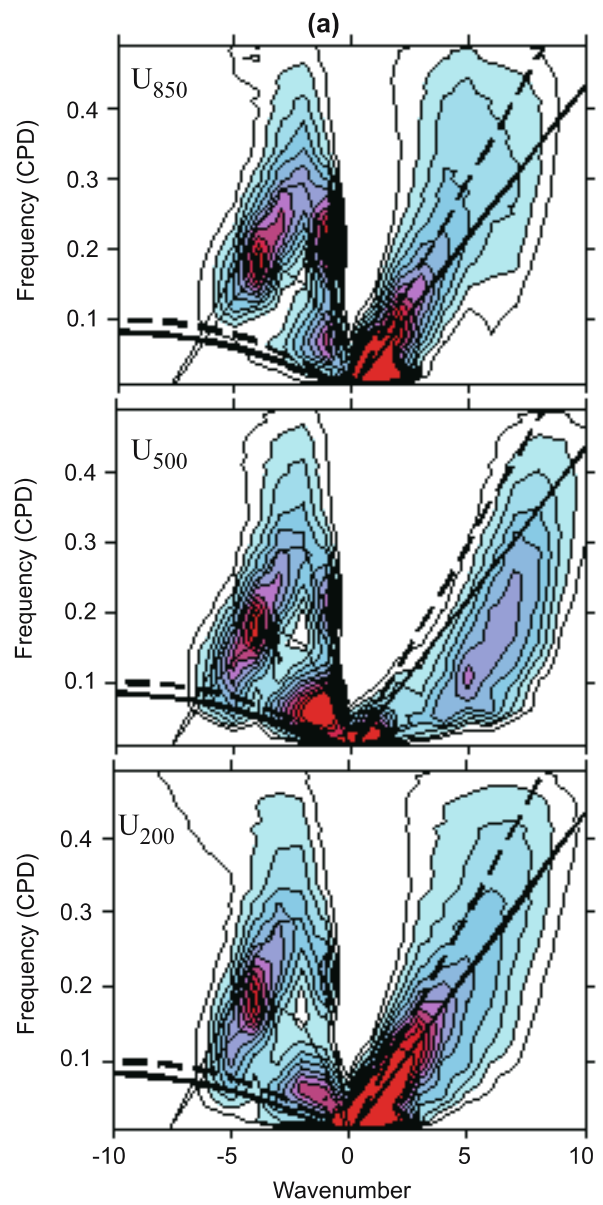

(b)

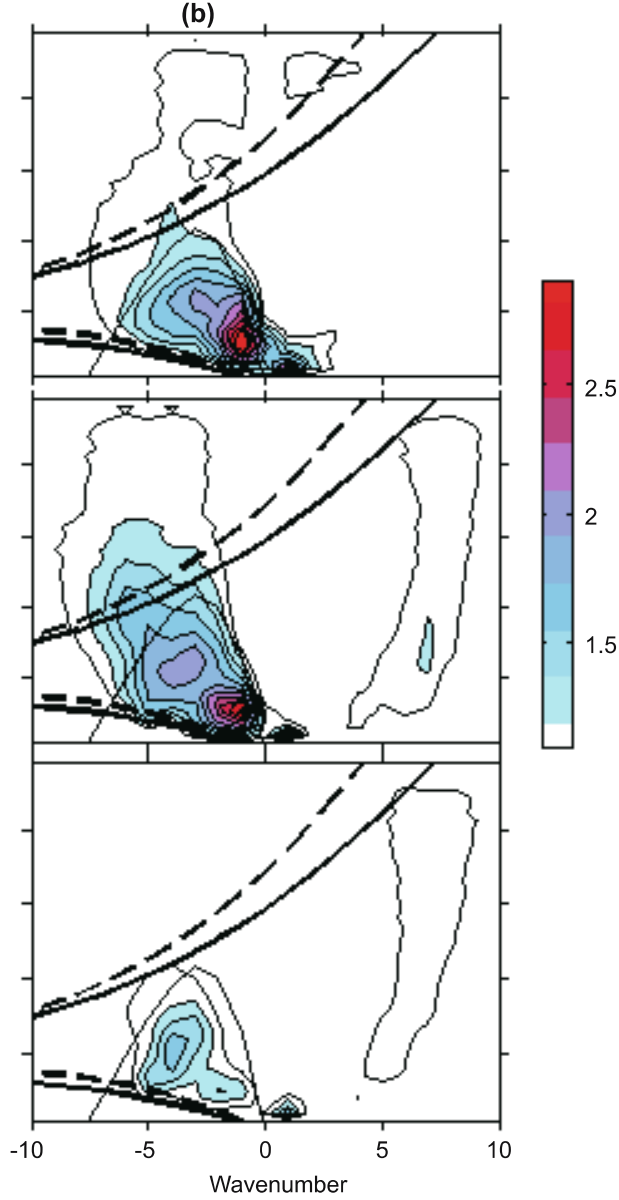

Figure 4. Same as figure 2 but for $U_{850}, U_{500}$ and $U_{200}$. (a) Symmetric part and (b) antisymmetric part. Thick lines represent theoretical dispersion curves for $h=40 \mathrm{~m}$ (solid) and $75 \mathrm{~m}$ (dashed) and thin lines for Rossby-Haurwitz modes ( $n=1$ and $n=3$ in symmetric and $n=2$ in antisymmetric) for $h=10 \mathrm{~km}$ and a zonal mean wind of $-15 \mathrm{~m} \mathrm{~s}^{-1}$.

bringing out the seasonal preferences of different modes. For example, Kelvin mode is strongest during April and May and weakest during August and September. Wavenumbers 1 to 4 are prominent during the boreal winter months in EISV whereas mainly $k=1$ mode dominates during July, August and September (JAS). Rossby mode is strong during boreal winter and much weaker during July to September. TD type modes are stronger mainly during July to September (i.e., Indian summer monsoon period), and practically not seen during December and January.

Spectra of the antisymmetric part show less intensity compared to the symmetric part during all the months (figure 7b). Maximum observed intensity is in the EISV mode. Monthly variations in EISV activity in the antisymmetric part are similar to that in the symmetric part but with reduced intensity. The other dominant/significant mode observed is MRG mode, again having a strong seasonal dependence. This mode is weakest during February and strongest during April and October. There is also a large variation in the preferred frequencies and wavenumbers. For example, it has eastward propagating modes during January-March, whereas both eastward and westward modes are equally prominent during October-November.

Most of the features observed in figure 7 are in broad agreement with earlier results based on seasonal averages (taking May to October period as boreal summer and November to April as boreal winter). WK99 and Cho et al (2004) have observed the peaks in EISV and Rossby modes during boreal winter and TD type disturbances during boreal summer. The annual variation of different modes is given in Wheeler et al (2000) and Roundy and Frank (2004). Wavenumber-frequency filtering is done corresponding to the domain of each mode and the running mean variance of this filtered data over a window length corresponding to the highest time scale of the mode is used to quantify the mode. In case of Wheeler et al (2000) the variation at a single position where the variance of the mode peaks is given while Roundy and Frank (2004) presented global average variance over the tropics 

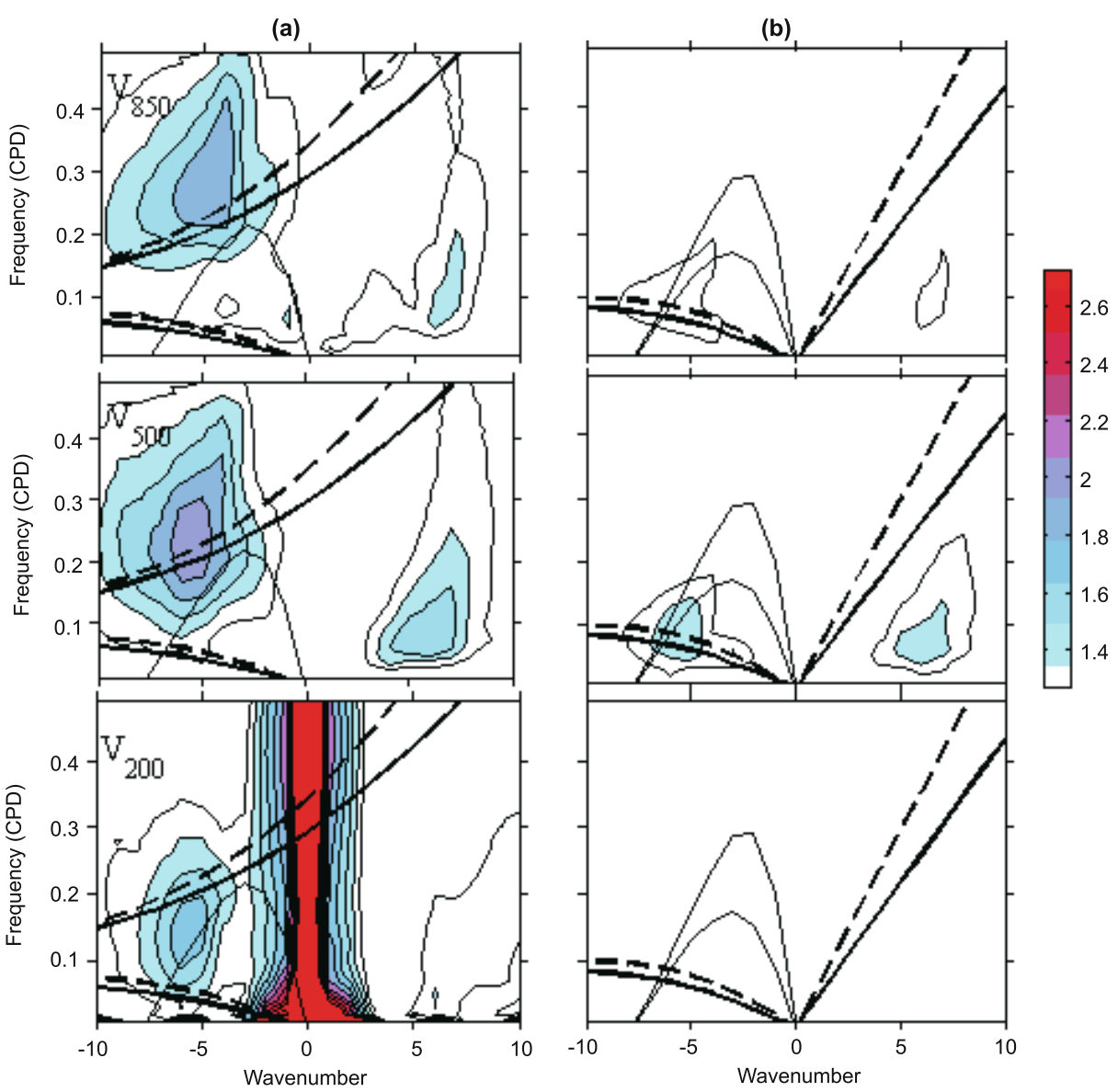

Figure 5. Similar to figure 4 for the meridional component of wind. The dispersion curves for MRG, $n=2$ Rossby and $n=2$ Rossby-Haurwitz modes are shown in the symmetric part and $n=1$ and $n=3$ Rossby-Haurwitz and Rossby modes in the antisymmetric part.
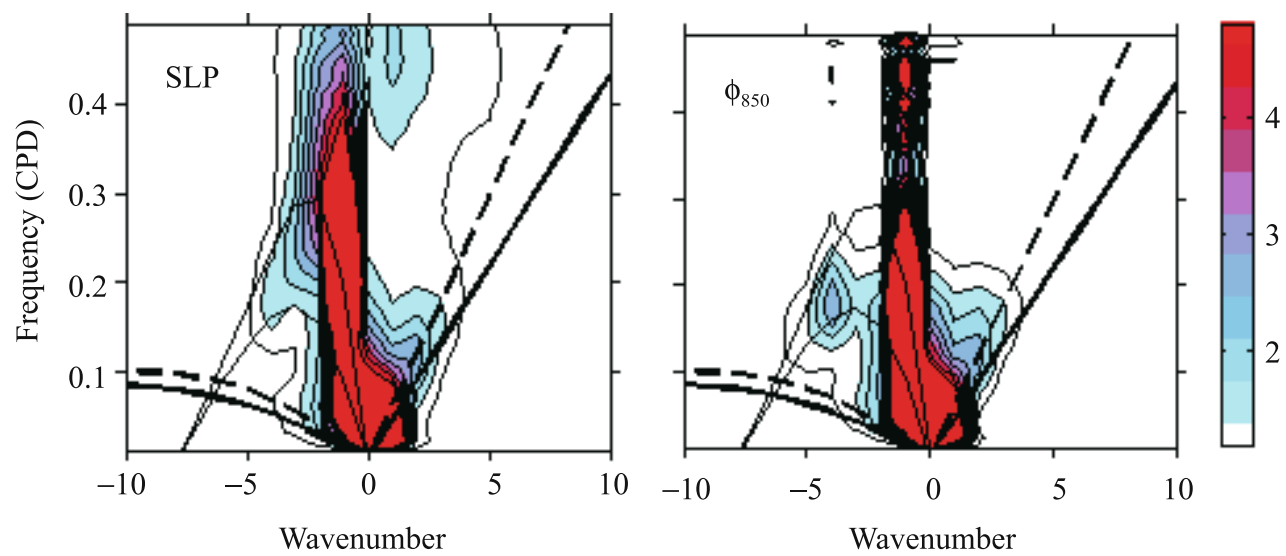

Figure 6. Symmetric part of the wavenumber-frequency spectra for SLP and $\phi_{850}$. Theoretical dispersion curves are same as in figure 4 .

$\left(20^{\circ} \mathrm{S}-20^{\circ} \mathrm{N}\right)$. They show similar annual variations as given in present study for all the modes. In contrast, the present method gives variations in monthly climatologies covering the entire spectral space of interest. Also variations in preferred wavenumbers and frequencies are presented.

\subsection{Meridional variations}

To study the meridional variations in the spectral characteristics, $10^{\circ}$ latitudinal belts are taken between $25^{\circ} \mathrm{S}$ and $25^{\circ} \mathrm{N}$, namely $25^{\circ}-15^{\circ} \mathrm{S}, 15^{\circ}-5^{\circ} \mathrm{S}$, $5^{\circ} \mathrm{S}-5^{\circ} \mathrm{N}, 5^{\circ}-15^{\circ} \mathrm{N}$ and $15^{\circ}-25^{\circ} \mathrm{N}$. The space time 

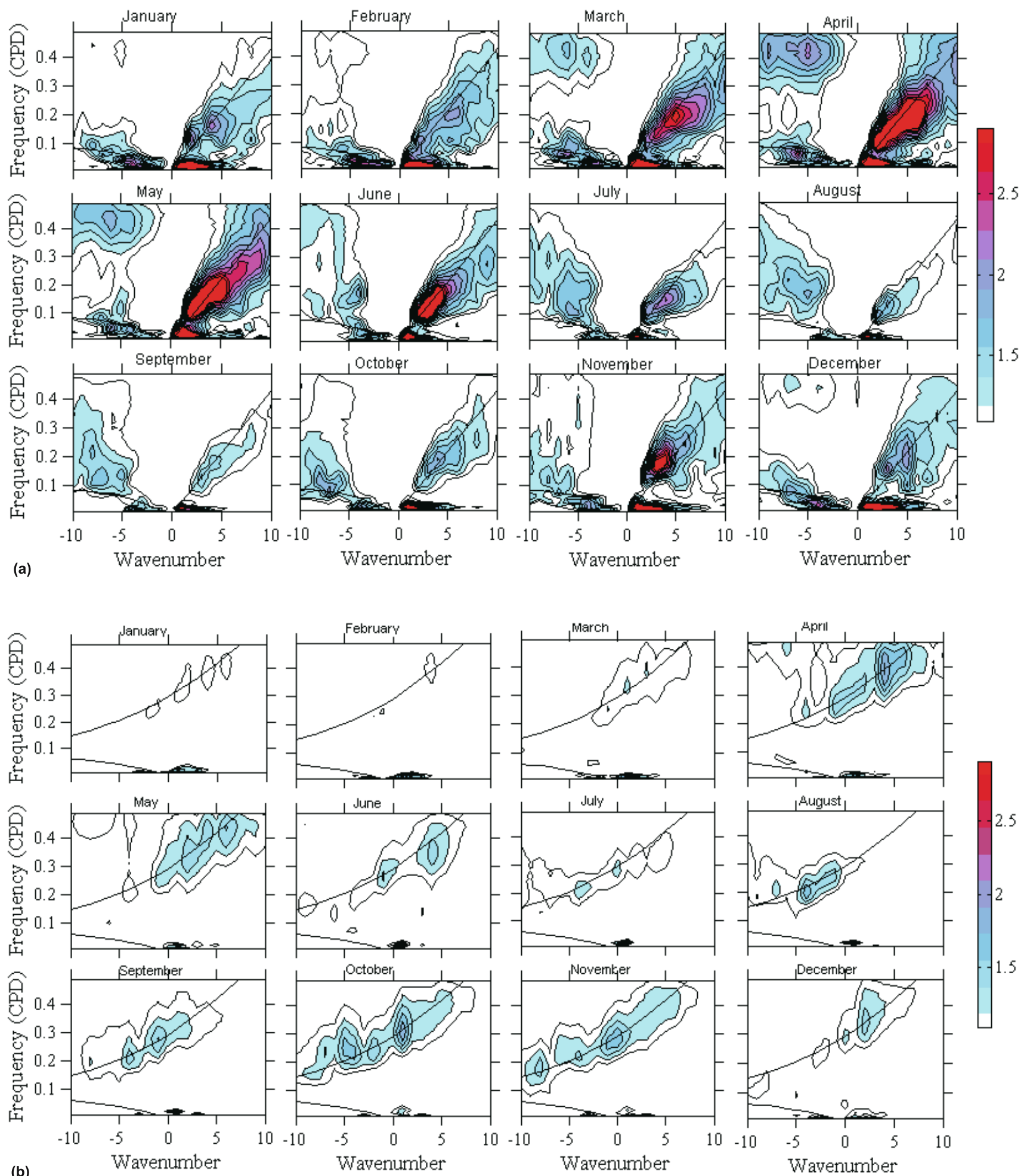

Figure 7. Monthly average spectra of OLR (a) symmetric part and (b) antisymmetric part. Theoretical lines for equatorial modes with $h=40 \mathrm{~m}$ are also shown.

spectra for each belt are calculated for the total OLR following procedures similar to that in the previous section. Here normalization is done with red-noise spectrum calculated from average of all the meridional belts so that the intensities for different latitudinal belts can be compared directly.
Figure 8 shows the time averaged spectra for different latitudinal belts. It is seen that the maximum intensity occurs in the equatorial region $\left(5^{\circ} \mathrm{S}-5^{\circ} \mathrm{N}\right)$ and in the belt $5^{\circ}-15^{\circ} \mathrm{N}$ for all the modes. This agrees with the similar observations made from OLR and precipitable water by Roundy 


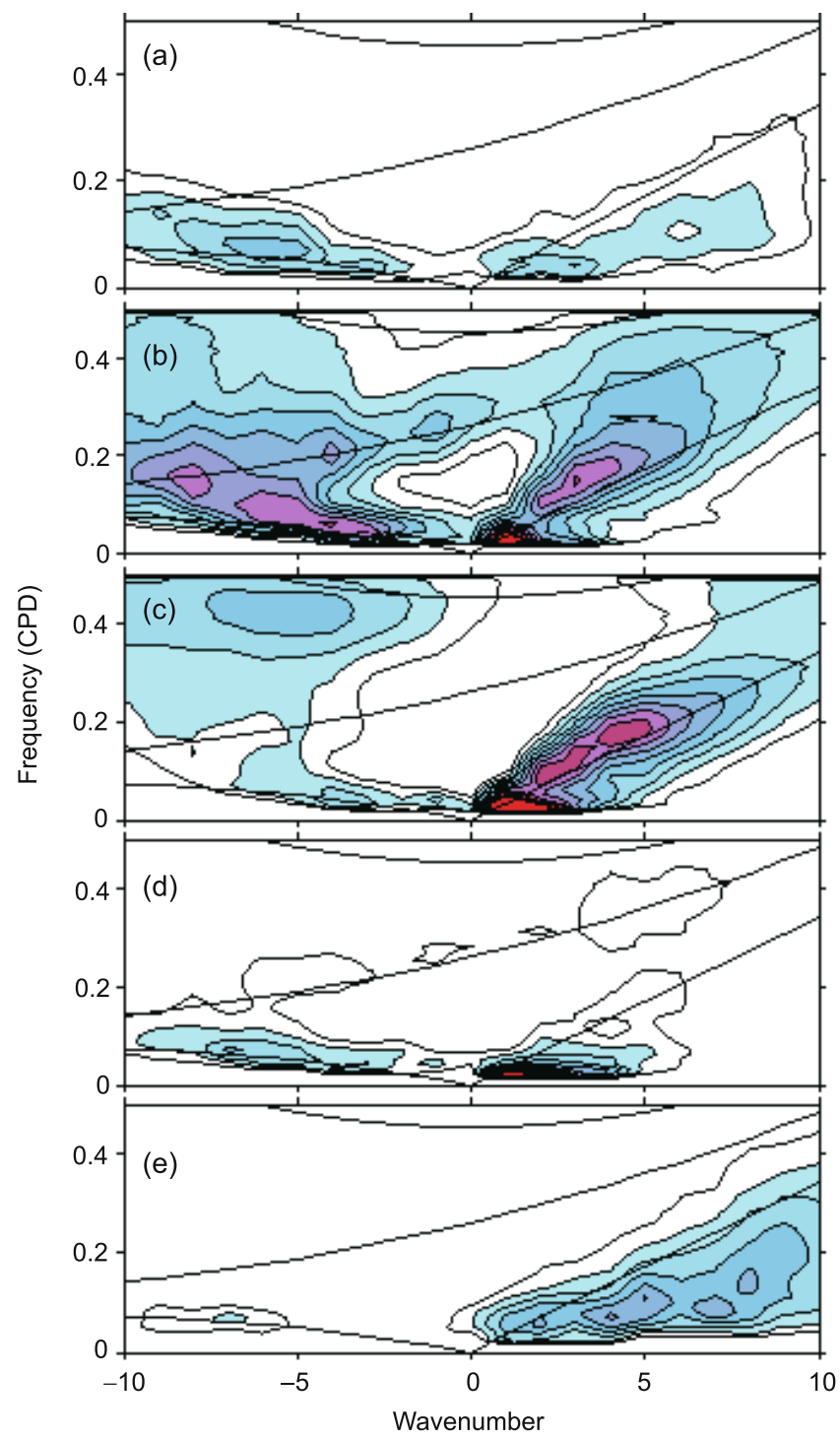

Figure 8. Wavenumber-frequency spectra of OLR for different latitudinal belts. (a) $15^{\circ}-25^{\circ} \mathrm{N}$; (b) $5^{\circ}-15^{\circ} \mathrm{N}$; (c) $5^{\circ} \mathrm{S}-5^{\circ} \mathrm{N}$; (d) $15^{\circ}-5^{\circ} \mathrm{S}$ and (e) $25^{\circ}-15^{\circ} \mathrm{S}$.

and Frank (2004). The EISV mode is most prominent in $5^{\circ} \mathrm{S}-5^{\circ} \mathrm{N}$ belt, and in a much weaker form up to $15^{\circ}-25^{\circ}$ belt on either side of equator as well. There is difference in spectral distribution in EISV mode between southern and northern hemispheres with more spread in frequency in north and more spread in wavenumber in the southern hemisphere. Beyond $15^{\circ}$ from the equator, the intensity corresponding to this mode is reduced. Kelvin mode has its peak in $5^{\circ} \mathrm{S}-5^{\circ} \mathrm{N}$ belt followed by the $5^{\circ}-15^{\circ} \mathrm{N}$ belt. Minimum activity is observed outside $15^{\circ}$ latitude from the equator. This is expected since the Kelvin wave is equatorially trapped. Rossby mode is present throughout the tropics. Rossby and MRG modes are stronger in $5^{\circ}-15^{\circ} \mathrm{N}$ belt rather than around the equator. This off-equatorial peak is also in agreement with previous studies (e.g.,
WK99; Masunaga 2007). TD type modes are not seen outside $15^{\circ}$ from the equator.

\section{Discussions and conclusions}

The wavenumber-frequency spectral characteristics of atmospheric variables in the tropics are presented. Spectra are obtained by the combined FFT and wavelet transforms. In the previous studies, main emphasis was given to variables connected with convection, namely OLR, precipitation and water vapour. It is shown here that some of the peaks observed in OLR corresponding to different theoretical modes in EWT are not as clear as in the case of SLP and $\phi_{850}$. Spectra of each variable have some characteristics different from others. Spectral characteristics of $U_{200}$ are more close to OLR compared to other fields and SLP is completely different from other variables with maximum intensity at lower wavenumbers and no significant power beyond wavenumbers \pm 5 . The zero wavenumber mode (i.e., zonal average) dominates SLP, $\phi_{850}$ and $V_{200}$. Any change in zonal average SLP implies change of mass in the atmospheric column above, and the mass adjustment has to take place in the meridional direction. In the case of SLP, the zero wavenumber mode has strong meridional connections with variations in tropics and high latitudes $\left(\sim 60^{\circ}\right.$ on either side of the equator) significantly correlated at zero time lag but out of phase (Kiranmayi and Bhat 2008). In the OLR spectrum, Kelvin wave peaks around 0.15 day $^{-1}$ frequency whereas in the case of zonal winds, the corresponding frequencies are lower. Spectra of zonal winds have peaks corresponding to Rossby-Haurwitz modes. OLR and $U_{200}$ spectrum show peaks corresponding to an equivalent depth of $40 \mathrm{~m}$, while zonal winds at $850 \mathrm{mb}$, SLP and $\phi_{850}$ have higher equivalent depth around $75 \mathrm{~m}$. EISV mode is present in the spectra of all the variables except $V_{850}$ and $V_{500}$.

Deep convection is associated with low level convergence. Therefore, we expected $850 \mathrm{hPa}$ divergence and OLR to have similar spectral patterns. Examination of figures 2 and 3 shows that the equivalent depths are comparable; however, there are some important differences too. In particular, the symmetric part of $850 \mathrm{hPa}$ divergence has a prominent zero wavenumber mode which is absent in OLR. Perhaps, this mode in divergence is connected with the Hadley circulation, which in the mean transports mass from mid latitudes to inter-tropical convergence zone at low levels. A prominent $k=0$ mode suggests that the zonal mean mass transport from extra tropics to tropics at low levels is not uniform in time but fluctuates (very likely to be associated with the deep 
convection in the tropical belt). There are other differences as well, in particular in the EISV regime in the symmetric part of the spectra. In the antisymmetric spectra, OLR has higher energy in westward propagating modes, whereas for the $850 \mathrm{hPa}$ divergence, more energy is in the eastward propagating modes.

It is observed that, in the case of OLR almost all the modes vary even on monthly time scales. In general, intraseasonal variations are stronger in the northern hemisphere compared to that in the southern hemisphere.

In Kelvin waves (Gill 1982), meridional component of velocity is zero and so is zonal component at mid levels for baroclinic mode one. The $U_{500}$ spectrum does show that in the spectral region corresponding to Kelvin wave, there is no significant power. Similarly, Kelvin mode is absent in meridional winds. These features are correctly reflected in the spectral analysis of $U$ and $V$. However, there is a region shifted to the right on wavenumber axis with significant energy in both meridional winds and $U_{500}$. Nature of this mode needs to be understood. Another point that needs further investigation is, why some variables have $40 \mathrm{~m}$ equivalent depth while others $75 \mathrm{~m}$.

To end, the main conclusions are:

- Winds, pressure, geopotential height show spectral characteristics different from that of OLR.

- OLR has peaks corresponding to an equivalent depth of $40 \mathrm{~m}$, whereas it is $75 \mathrm{~m}$ for horizontal components of wind velocity except at $200 \mathrm{hPa}$, SLP and $\phi_{850}$.

- Rossby-Haurwitz modes are observed in zonal winds and to some extent in SLP and meridional winds.

- For OLR data, all the equatorial modes show a certain degree of seasonality.

\section{Acknowledgements}

The NCEP reanalysis data and the interpolated OLR data are provided by NOAA/OAR/ESRL PSD, Boulder, Colorado, USA, and accessed from the website http://www.cdc.noaa.gov/. ERA-40 data is downloaded from ECMWF site www. ecmwf.int. This work was partially supported by grants from the Department of Science and Technology, New Delhi and the Ministry of Earth Sciences, New Delhi. We thank the two anonymous referees for their critical comments and useful suggestions.

\section{References}

Cho H K, Bowman K P and North G R 2004 Equatorial waves including the Madden-Julian oscillation in TRMM rainfall and OLR data; J. Climate 17 4387-4406.
Daubechies I 1990 The wavelet transform time-frequency localization and signal analysis; IEEE Trans. Inform. 36 961-1004.

Emery W J and Thomson R E 2001 Data Analysis Methods in Physical Oceanography; Springer, pp. 514-549.

Gill A E 1982 Atmosphere - Ocean Dynamics (San Diego: Academic Press) 662 pp.

Gu G and Zhang C 2001 A spectrum analysis of synoptic scale disturbances in the ITCZ; J. Climate 14 $2725-2739$.

Hendon H H and Wheeler M 2008 Some space-time spectral analyses of tropical convection and planetary-scale waves; J. Atmos. Sci. 65 2936-2948.

Kaiser G 1994 A Friendly Guide to Wavelets, Birkhäuser, $300 \mathrm{pp}$.

Kalnay E, Kanamitsu M, Kistler R, Collins W, Deaven D, Gandin L, Iredell M, Saha S, White G, Woollen J, Zhu Y, Chelliah M, Ebisuzaki W, Higgins W, Janowiak J, Mo K C, Ropelewski C, Wang J, Leetma A, Reynolds R, Jenne R and Joseph D 1996 The NCEP/NCAR 40-year Reanalysis project; Bull. Amer. Meteor. Soc. 77 $437-471$.

Kailash S V and Narasimha R 2000 Quasi-cycles in monsoon rainfall by wavelet analysis; Curr. Sci. 78 592-595.

Kasahara A 1980 Effect of zonal flows on the free oscillations of a barotropic atmosphere; J. Atmos. Sci. 37 917-929.

Kiranmayi L and Bhat G S 2008 Quasi-periodic, global oscillations in sea level pressure on intraseasonal time scales; Clim. Dyn. 31 doi:10.1007/s00382-008-0413-7.

Lau W K M and Waliser D E 2005 Intraseasonal Variability in the Atmosphere-Ocean Climate System (Chichester, UK: Springer) $436 \mathrm{pp}$.

Lawrence D M and Webster P J 2001 Interannual variations of the intraseasonal oscillation in the south Asian summer monsoon region; J. Climate 14 2910-2922.

Liebmann B and Smith C A 1996 Description of a complete (interpolated) OLR dataset; Bull. Amer. Meteor. Soc. $\mathbf{7 7}$ 1275-1277.

Madden R A 1978 Further evidence of traveling planetary waves; J. Atmos. Sci. 35 1605-1618.

Madden R A and Julian P R 1971 Description of a 40-50 day oscillation in the zonal wind in the tropical Pacific; J. Atmos. Sci. 29 1109-1123.

Masunaga H 2007 Seasonality and regionality of the Madden-Julian oscillation, Kelvin wave, and equatorial Rossby wave; J. Atmos. Sci. 64 4400-4416.

Matsuno T 1966 Quasi-geostropic motions in the equatorial area; J. Meteor. Soc. Japan 44 25-43.

Rao R M and Bopardikar A S 1998 Wavelet transforms: Introduction to theory and applications; Addison-Wesley.

Roundy P E and Frank W M 2004 A climatology of waves in the equatorial region; J. Atmos. Sci. 61 2105-2132.

Shanker A P and Nanjundiah R S 2003 Morlet wavelet analysis of tropical convection over space and time: Study of poleward propagation of intertropical convergence zone (ITCZ); Geophys. Res. Lett. 31 doi:10.1029/2003GL018150.

Slingo J M, Sperber K R, Boyle J S, Ceron J P, Dix M, Dugas B, Ebisuzaki W, Fyfe J, Gregory D, Gueremy J F, Hack J, Harzallah A, Inness P, Kitoh A, Lau W K M, McAvaney B, Madden R, Matthews A, Palmer T N, Park C K, Randall D and Renno N 1996 Intraseasonal oscillations in 15 atmospheric general circulation models: Results from an AMIP diagnostic subproject; Clim. Dyn. $12325-357$.

Teng H and Wang B 2003 Interannual Variations of the Boreal Summer Intraseasonal Oscillation in the Asian-Pacific Region; J. Climate 16 3572-3584. 
Torrence C and Compo G P 1998 A practical guide to wavelet analysis; Bull. Amer. Meteor. Soc. 79 61-78.

Uppala S M, Kallberg P W, Simmons A J, Andrae U, Da Costa Bechtold V, Fiorino M, Gibson J K, Haseler J, Hernandez A, Kelly G A, Li X, Onogi K, Saarinen S, Sokka N, Allan R P, Andersson E, Arpe K, Balmaseda M A, Beljaars A C M, Van De Berg L, Bidlot J, Bormann N, Caires S, Chevallier F, Dethof A, Dragosavac M, Fisher M, Fuentes M, Hagemann S, Hólm E, Hoskins B J, Isaksen L, Janssen P A E M, Jenne R, Mcnally A P, Mahfouf J F, Morcrette J J, Rayner N A, Saunders R W, Simon P, Sterl A, Trenberth K E, Untch A, Vasiljevic D, Viterbo P, Woollen J 2005 The ERA-40 re-analysis; Quart. J. Roy. Meteor. Soc. 131 2961-3012.

Wallace J M and Kousky V E 1968 Observational evidence of Kelvin waves in the tropical stratosphere; J. Atmos. Sci. 25 280-292.

Wang B 2005 Theory (Intraseasonal Variability in the Atmosphere-Ocean Climate System), (eds) Lau W K M and Waliser D E (Chichester, UK: Springer) pp. 307-317.

Wheeler M C 2002 Tropical meteorology: Equatorial waves. (Encyclopedia of Atmospheric Sciences), (eds)
Holton J, Curry J and Pyle J, Academic Press, $2313-2325$.

Wheeler M and Kiladis G N 1999 Convectively coupled equatorial waves: Analysis of clouds and temperature in the wavenumber-frequency domain; J. Atmos. Sci. 56 374-399.

Wheeler M and Hendon H H 2004 An all-season realtime multivariate MJO index: Development of an index for monitoring and prediction; Mon. Wea. Rev. 132 1917-1932.

Wheeler M, Kiladis G N and Webster P J 2000 Large scale dynamical fields associated with convectively coupled equatorial waves; J. Atmos. Sci. 57 613-640.

Wong M L M 2009 Wavelet analysis of the convectively coupled equatorial waves in the wavenumber-frequency domain; J. Atmos. Sci. 66 209-212.

Yanai M and Maruyama T 1966 Stratospheric wave disturbances propagating over the equatorial Pacific; J. Meteor. Soc. Japan. 44 291-294.

Zhang C 2005 Madden-Julian oscillations; Rev. Geophys. $431-36$.

Zhang C and Dong M 2004 Seasonality of Madden Julian Oscillation; J. Climate 17 3169-3180. 